\title{
Olfactory senses linked to metabolism
}

New research demonstrates that the perception of food via the olfactory senses can alter energy homeostasis in mice. Andrew Dillin, Jens Brüning (co-corresponding authors) and colleagues found that animals lacking olfactory sensory perception ate the same amount as animals that could smell, but did not gain fat mass when fed a high-fat diet.

"In prior studies, the sense of smell has been shown to affect ageing in worms and flies," explains Dillin. "In our study, we wanted to investigate what impact the perception of food via smell had on the assimilation of calories." How well mice are fed directly alters olfactory sensitivity; when satiated, mice have a reduced sense of smell but in the fasted state olfactory senses are stimulated. These data suggest a link between peripheral metabolism and olfactory sensory neurons.

In mice, olfactory sensory neurons can indirectly communicate with the hypothalamus via the centres of the olfactory cortex. Following olfactory bulb stimulation, neuronal signals are transmitted to the olfactory cortex, which innervates the hypothalamus through the olfactory tubercle. Dillin, Brüning and co-workers hypothesized that olfactory signals might alter systemic metabolism in response to anticipated food intake by simulating homeostatic neurons in the hypothalamus.

To investigate the role of olfactory senses in the control of whole-body homeostasis, Dillin and colleagues generated mice that express diphtheria toxin receptor in ophthalmic sensory neurons. They conditionally ablated the sense of smell in adult mice by administering diphtheria toxin intraperitoneally at 7 weeks of age. During the first 7 weeks of life, the mice had a functioning olfactory system and so were able to learn how to locate food. Ablating their sense of smell did not affect this learned behaviour or affect their ability to eat. To assess whether the ablation of olfactory sensory neurons altered body weight, the team examined the effects of hyposmia (the reduced ability to smell and to detect odours) in lean mice and mice with diet-induced obesity.

The team found that the ablation of smell resulted in an increase in energy expenditure through increased lipolysis and the stimulation of thermogenesis in brown adipose tissues. Interestingly, in severely obese mice on a high-fat diet, the increased energy exposure resulted in a reduction in adiposity, an effect that occurred after just one injection of diphtheria toxin. Repeated exposure to diphtheria toxin reduced adiposity but did not alter lean mass even though mice continued to eat the high-fat diet.

The team then investigated whether hormonal signalling can alter the perception of smell. mRNA expression of endocrine hormone receptors, such as the insulin-like growth factor 1(IGF1) receptor, is high in the olfactory system. For these experiments, Brüning and colleagues developed a mouse model with reduced IGF1 receptor expression in the olfactory epithelium. The data show that these mice have a heightened smell perception, increased adiposity, hyperleptinaemia, hyperinsulinaemia and insulin resistance.

These data suggest that losing the sense of smell can protect mice from the adverse effects of eating a highfat diet. Furthermore, the authors' findings show that the ablation of the olfactory sensory neurons can reverse diet-induced obesity in mice. Thus, the authors have described a novel pathway for how the olfactory system can control whole-body energy homeostasis.

Alan Morris

...the authors' findings show that the ablation of the olfactory sensory neurons can reverse diet-induced obesity in mice...
ORIGINAL ARTICLE Riera, C. E. et al. The sense of smell impacts metabolic health and obesity. Cell Metab. 26, 198-211.e5 (2017) 